\title{
Análise das práticas de biossegurança dos profissionais atuantes em unidade de terapia intensiva: Estudo transversal
}

Analysis of biosafety practices of professionals working in the intensive care unit: Cross-sectional study

Análisis de prácticas de bioseguridad de profesionales que actúan en unidad de cuidados intensivos: Estudio cruzado

\section{Resumo}

O objetivo deste estudo é investigar o emprego das práticas de biossegurança pelos profissionais de saúde atuantes em uma UTI de um hospital público de Teresina-PI. Trata-se de um estudo transversal, aprovado pelo comitê de ética (2.583.723), realizado em um hospital estadual de Teresina-PI, mediante aplicação de questionários aos profissionais de saúde da UTI. Os dados foram analisados através dos programas SPSS 17.0 e BioEstat 5.0, com aplicação dos testes Qui-quadrado, Wilcoxon e Kruskal Wallis. Foram considerados significativos valores com p<0,05. Foram entrevistados 26 profissionais, com predomínio dos que possuíam acima de 10 anos de formados $(53,8 \%$, p <0,006, $\mathrm{n}=14)$, com mais de 3 anos de experiência profissional $(76,9 \%, \mathrm{n}=20, \mathrm{p}=0,001 * * *)$ e acima de 3 anos atuando no referido hospital $(76,9 \%, \mathrm{n}=20, \mathrm{p}<0,001 * * *) .96,2 \%$ relatam usar os EPIs, com restrição de uso para propés $(92,3 \%)$ e óculos de proteção $(84,6 \%) .18(69,2 \%)$ referem serem cobrados o conhecimento das normas de biossegurança pelo hospital, divergindo com a percepção de conhecimento e atualização dos mesmos. Há uma deficiência do conhecimento dos profissionais no conhecimento e no rigor das práticas de biossegurança.

Palavras-chave: Unidade de terapia intensiva; Infecção; Biossegurança.

\begin{abstract}
The aim of this study is to investigate the use of biosafety practices by health professionals working in an ICU of a public hospital in Teresina-PI. This is a cross-sectional study, approved by the ethics committee $(2,583,723)$, conducted at a state hospital in Teresina-PI, using questionnaires to ICU health professionals. The data were analyzed using the SPSS 17.0 and BioEstat 5.0 programs, using the Chi-square, Wilcoxon and Kruskal Wallis tests. Values with $\mathrm{p}<0.05$ were considered significant. 26 professionals were interviewed, with a predominance of those with more than 10 years of training $(53.8 \%, p<0.006, n=14)$, with more than 3 years of professional experience $(76.9 \%, n=20$, $\mathrm{p}=0.001 * * *)$ and older than 3 years working at the referred hospital $(76.9 \%, \mathrm{n}=20, \mathrm{p}<0.001 * * *) .96 .2 \%$ reported using PPE, with use restrictions for propes (92.3\%) and goggles (84.6\%). 18 (69.2\%) reported that they were charged with the knowledge of biosafety standards by the hospital, differing with the perception of knowledge and updating of them. There is a deficiency in the knowledge of professionals in the knowledge and rigor of biosafety practices.
\end{abstract}

Keywords: Intensive care unit; Infection; Biosafety.

\section{Resumen}

El objetivo de este estudio es investigar el uso de prácticas de bioseguridad por profesionales de la salud que trabajan en una UCI de un hospital público de Teresina-PI. Se trata de un estudio transversal, aprobado por el comité de ética $(2,583,723)$, realizado en un hospital estatal de Teresina-PI, mediante cuestionarios a profesionales de la salud de 
UCI. Los datos se analizaron mediante los programas SPSS 17.0 y BioEstat 5.0, utilizando las pruebas Chi-cuadrado, Wilcoxon y Kruskal Wallis. Los valores con p $<0,05$ se consideraron significativos. Se entrevistaron 26 profesionales, con predominio de aquellos con más de 10 años de formación $(53,8 \%, \mathrm{p}<0,006, \mathrm{n}=14)$, con más de 3 años de experiencia profesional $(76,9 \%, \mathrm{n}=20, \mathrm{p}=0,001 * * *)$ y mayores de 3 años laborando en el hospital de referencia $(76,9 \%, \mathrm{n}=20, \mathrm{p}<0,001 * * *)$. El 96,2\% reportó usar EPP, con restricciones de uso de propes $(92,3 \%)$ y gafas $(84,6 \%)$. $18(69,2 \%)$ refirieron estar a cargo del conocimiento de los estándares de bioseguridad por parte del hospital, diferenciándose en la percepción del conocimiento y actualización de los mismos. Existe una deficiencia en el conocimiento de los profesionales en el conocimiento y rigor de las prácticas de bioseguridad.

Palabras clave: Unidad de cuidados intensivos; Infección; Bioseguridad.

\section{Introdução}

A Unidade de Terapia Intensiva (UTI) é uma unidade de cuidados que visa promover suporte ao paciente crítico com agilidade e segurança por meio da oferta de suporte avançado e atenção continuada, fazendo uso de recursos de alta tecnologia (Santuzzi et al., 2013). É avaliada como um espaço higienizado e de alto controle endêmico, no entanto, apesar da intensa vigilância, dada às patogenias ali tratadas, o risco de contaminação hospitalar é permanente (Barros et al., 2016), fazendo-se necessário o uso de estratégias que visem o controle das infecções, tais como os protocolos de biossegurança (Silva \& Rodrigues, 2015).

O conceito de biossegurança começou a ser abordado na década de 70 nos Estados Unidos, quando a comunidade científica iniciou a discussão sobre os impactos da engenharia genética na sociedade (Almeida \& Albuquerque, 2000). De acordo com a Agência Nacional de Vigilância Sanitária (Anvisa, 2005), biossegurança é um conjunto de ações destinadas a prevenir, controlar, reduzir ou eliminar riscos inerentes às atividades que possam comprometer a saúde humana.

As normas de biossegurança, no Brasil, são descritas a partir do Decreto de Lei 6.367 de 19 de outubro de 1976, no qual ressalta a importância da atenção que deve ser dada às medidas de segurança no âmbito hospitalar, em especial, na UTI (Correa \& Donato, 2007). Todavia, mesmo sabendo da importância da biossegurança, observa-se uma certa dificuldade na aplicação destas na prática hospitalar (Nishide et al., 2004; Talhaferro et al., 2008).

Em 2020, dada a pandemia do SARS-CoV-2, no qual por se caracterizar como um vírus com alto poder de contágio, obrigou que todos as pessoas, sejam elas profissionais de saúde ou não, fizessem o uso das normas de biossegurança, pautadas na rigidez da higienização e no uso de equipamentos de proteção individual (EPIs), visando a não disseminação viral em nível social e a piora do quadro clínico dos pacientes criticamente enfermos internados nos hospitais, bem como na proteção dos próprios profissionais (World Health Organization, 2020). Contudo, sabe-se que independente de uma pandemia, os hospitais, em especial as UTIs, são focos de inúmeros patógenos, capazes de propagar ou gerar novas infecções, que culminam na piora do quadro clínico dos pacientes, ou mesmo expõem os profissionais e familiares à infecção (Pinto et al., 2005; Aguiar et al., 2008).

Dentro desse contexto, sabendo da alta incidência de infecções nas UTIs, que culminam na piora do quadro clínico do paciente, conduzindo ao aumento de tempo de internação e/ou até a morte dos mesmos, bem como a exposição dos profissionais aos riscos de contaminação, busca-se enfatizar a importância das práticas de biossegurança e o uso das EPIs. Logo, partindo disso, buscou-se investigar o emprego dos EPIs e das práticas de biossegurança pelos profissionais de saúde atuantes em uma UTI de um hospital público de Teresina-PI.

Diante dos riscos de infecções apresentados nas UTIs, este estudo traz a reflexão sobre a importância dos cuidados sobre os riscos de infecção em pacientes críticos, bem como dos profissionais de saúde, enfatizando a importância das normas de biossegurança a nível hospitalar, em especial, nas UTIs. 


\section{Metodologia}

\subsection{Princípios éticos}

A presente pesquisa foi realizada de acordo com as diretrizes para pesquisa envolvendo humanos preconizadas pela Resolução do Conselho Nacional de Saúde 466/12, sendo a mesma submetida à apreciação do Comitê de Ética em Pesquisa com Seres Humanos do Centro Universitário UNIFACID e aprovada sem restrições sob o número de parecer 2.583.723.

\subsection{Desenho e participantes}

Trata-se de um estudo transversal, com abordagem quantitativa, realizado em uma UTI de um Hospital Estadual, localizado na cidade de Teresina- PI, no período de agosto a outubro de 2019. A população acessível deste estudo foi composta dos profissionais que fazem parte da equipe multidisciplinar plantonista atuante na UTI do referido hospital, contemplando: enfermeiras, fisioterapeutas, técnicas de enfermagem e médicos, distribuídos nos turnos matutino, vespertino e noturno.

A amostra foi selecionada por conveniência a partir da organização dos plantões e dos profissionais contemplados obtidos junto à direção do hospital. Foram incluídos os profissionais que tivessem contato com o ambiente da UTI, pacientes e equipamentos, que atuassem em pelo menos dois plantões de carga-horária mínima de 12 horas cada no turno observado, sendo excluídos os profissionais que trabalham em outros setores do hospital e que solicitaram a sua retirada do estudo.

Todos os voluntários validaram a sua participação na pesquisa por meio da assinatura no termo de consentimento livre esclarecido (TCLE), após a explanação da finalidade do estudo, bem como, assegurado o seu direito à privacidade, confidencialidade e de retirar seu consentimento sem que lhe ocasionasse algum prejuízo.

\subsection{Instrumentos de Avaliação}

Os dados foram coletados por meio de entrevista e observação, registrados manualmente, via utilização de um roteiro estruturado, composto por 15 questões, com tempo médio de reposta 30 minutos, aplicadas por um único pesquisador devidamente treinado e apto para aplicação do questionário e realizar as observações. O questionário aplicado para coleta dos dados foi elaborado pelos autores com base nas questões norteadoras. O questionário foi aplicado no ambiente hospitalar, em área externa à UTI. A primeira parte contemplou a caracterização dos participantes segundo o gênero, faixa etária, categoria profissional, tempo de formação e o uso dos EPIs, seguido da análise do conhecimento a respeito das normas de biossegurança em ambiente hospitalar. Já a segunda parte foi destinada ao registro dos procedimentos realizados por cada membro da equipe durante a prestação do cuidado, tais como a higienização das mãos, intervenções junto aos pacientes, uso ou não dos EPIs.

\subsection{Análise Estatística}

Os dados referentes aos questionários foram tabulados por meio do programa Microsoft Office Excel ${ }^{\circledR}$ versão 1.0 e realizada a análise estatística através dos programas Statistical Package for the Social Sciences - SPSS, versão 17.0 e BioEstat 5.0 por meio da aplicação dos testes: Qui-quadrado, Wilcoxon e Kruskal Wallis. Foram considerados significativos valores com $\mathrm{p}<0,05$. Os dados foram expressos em tabelas e figuras.

\section{Resultados}

Participaram do estudo 26 profissionais, distribuídos entre 16 técnicos de enfermagem $(61,5 \%, \mathrm{p}=0,53), 5$ fisioterapeutas $(19,3 \%, \mathrm{p}=0,53), 3$ enfermeiras $(11,5 \%, \mathrm{p}=0,53)$ e 2 médicos $(7,7 \%, \mathrm{p}=0,53)$. Em sua totalidade eram do sexo feminino, com média de idade $\pm 38,5$ (21- 56) anos. Prevaleceram profissionais com tempo de formação maior que 10 anos $(53,8, \mathrm{n}=14, \mathrm{p}<0,006)$, com mais de 3 anos de experiência profissional $(76,9 \%, \mathrm{n}=20, \mathrm{p}=0,001)$ e acima de 3 anos atuando no referido hospital $(76,9 \%, \mathrm{n}=20, \mathrm{p}<0,001)$, como mostra a Tabela 1 . 
Tabela 1 - Perfil da amostra de profissionais atuantes em Unidade de Terapia Intensiva de Hospital Estadual, Teresina, 2019, $\mathrm{n}=26$.

\begin{tabular}{clccc}
\hline Variável & Categoria & $\mathbf{n}$ & $\mathbf{( \% )}$ & $\boldsymbol{p}$ valor \\
\hline Profissão & Médico & 2 & 7,7 & \\
& Enfermeiro & 3 & 11,5 & \multirow{2}{*}{$0,002^{* * * *}$} \\
& Fisioterapeuta & 5 & 19,3 & \\
& Técnico de enfermagem & 16 & 61,5 & \\
& TOTAL & $\mathbf{2 6}$ & $\mathbf{1 0 0}$ & \\
\hline Tempo de formação & Até 2 & 4 & 15,4 & \\
(anos) & 2 a 10 & 8 & 30,8 & $0,006^{* * * *}$ \\
& $>10$ & 14 & 53,8 & \\
& TOTAL & $\mathbf{2 6}$ & $\mathbf{1 0 0}$ & \\
\hline Tempo de experiência & Até 2 & 3 & 11,5 & \\
profissional & Entre 2 a 3 & 3 & 11,6 & $<0,001^{* * *}$ \\
(anos) & $>3$ & 20 & 76,9 & \\
& TOTAL & $\mathbf{2 6}$ & $\mathbf{1 0 0}$ & \\
\hline Tempo de trabalho na & De 1 a 2 & 4 & 15,4 & \\
instituição & Entre 2 e 3 & 2 & 7,7 & $<0,001^{* * *}$ \\
(anos) & $>3$ & 20 & 76,9 & \\
& TOTAL & $\mathbf{2 6}$ & $\mathbf{1 0 0}$ & \\
\hline
\end{tabular}

Legenda: $\mathrm{N=}$ Número de participantes; $\%=$ percentual; ${ }^{*} p$ valor estatisticamente significante (Teste de Qui-quadrado). Fonte: Autores (2019).

Em relação à disponibilidade dos EPIs aos profissionais de saúde da UTI, a Figura 1 mostra que todos os participantes $(100 \%, \mathrm{n}=26)$ referiram ter acesso à touca, avental, luvas e máscaras, tendo acesso limitado quanto ao uso dos propés $(92,3 \%$, n=24) e aos óculos de proteção $(84,6 \%, n=22)$.

Figura 1 - Equipamentos de proteção individual disponíveis aos profissionais da Unidade de Terapia Intensiva. Teresina, $2019, \mathrm{n}=26$.

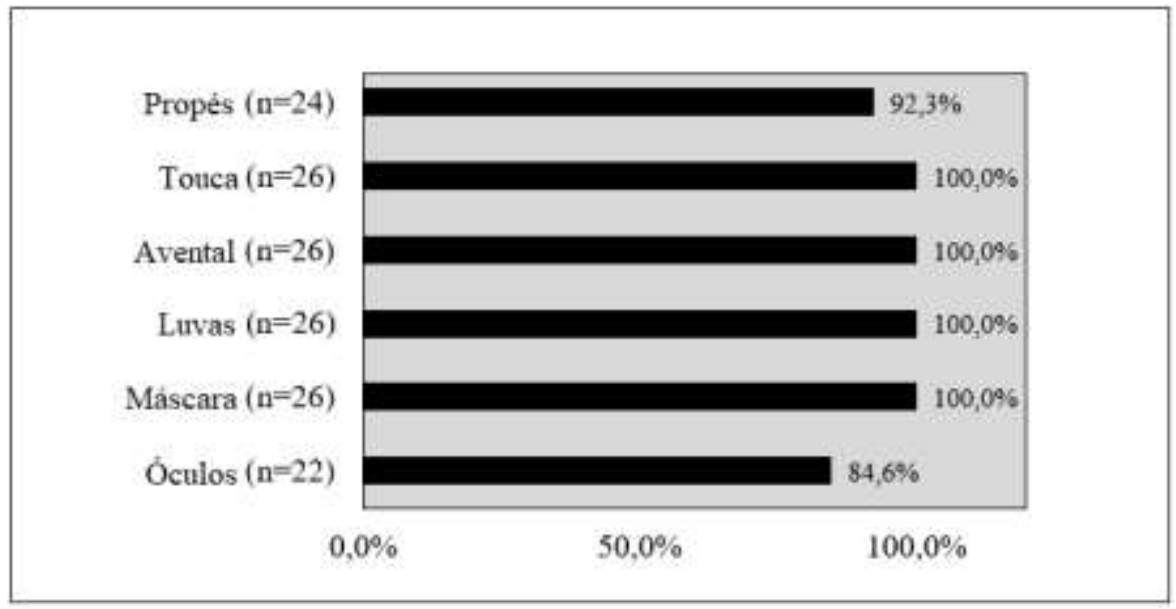

Legenda: N= Número de participantes; *p valor estatisticamente significante (Teste de Wilcoxon). Fonte: Autores (2019).

A Tabela 2 mostra os procedimentos de biossegurança realizados pelos profissionais: a frequência do uso dos EPIs nas intervenções; uso de vestimenta adequada e a frequência na troca entre os plantões; e a higienização das mãos. Dentre os avaliados, 96,2\% ( $\mathrm{n}=25)$ fizeram o uso dos EPIs, no entanto, a utilização dos mesmos não ocorreu em todas as intervenções, sendo priorizado o uso de todos em intervenções invasivas $(23,1 \%, n=6)$. Quanto às vestimentas, a troca foi referida como uma 
única vez, na entrada e na saída do plantão $(92,3 \%)$, enquanto que, $100 \%(\mathrm{n}=26)$ dos entrevistados referiram realizar a higienização das mãos acima de 5 vezes ou sempre antes e após realizado alguma intervenção no paciente.

Tabela 2 - Distribuição de respostas dos profissionais em questionário sobre práticas de biossegurança em Unidade de Terapia Intensiva. Teresina, 2019, $\mathrm{n}=26$.

\begin{tabular}{clcc}
\hline Variável & Categoria & $\mathbf{n}$ & $\mathbf{( \% )}$ \\
\hline & Sim* & 25 & 96,2 \\
Utiliza os EPI na UTI & Não & 1 & 3,8 \\
& As vezes & 0 & 0,0 \\
& TOTAL & $\mathbf{2 6}$ & $\mathbf{1 0 0}$ \\
\hline Frequência com que utiliza & Sempre ao realizar intervenções* & 20 & 76,9 \\
EPI & Apenas em intervenções invasivas & 6 & 23,1 \\
& TOTAL & $\mathbf{2 6}$ & $\mathbf{1 0 0}$ \\
\hline Frequência com que troca a & Não troca & 2 & 7,7 \\
roupa durante o plantão & 1 vez* & 24 & 92,3 \\
& TOTAL & $\mathbf{2 6}$ & $\mathbf{1 0 0}$ \\
\hline Frequência com que lava as & Mais de 5 vezes ou sempre antes de depois de & 26 & 100 \\
mãos (por turno) & intervenções* & 0 & 0,0 \\
& Abaixo de 5 vezes & $\mathbf{2 6}$ & $\mathbf{1 0 0}$ \\
\hline
\end{tabular}

Legenda: N= Número de participantes; \%= percentual; *conduta adequada (Teste de Wilcoxon). Fonte: Autores (2019).

O conhecimento das normas de biossegurança pelos profissionais entrevistados é apresentado na Tabela 3, na qual apenas 18 (69,2\%) referiram serem cobrados o conhecimento das normas de biossegurança no referido hospital. Observa-se que apesar dos profissionais serem cobrados, há uma contradição quanto ao conhecimento e/ou atualização demonstrados por eles. Destes apenas $2(7,7 \%)$ evidenciaram ler as normas de biossegurança frequentemente, $4(15,3 \%)$ confirmaram nunca terem lido, $6(23,1 \%)$ afirmam ter conhecimento das normas, porém, sem promover atualização ou que não lembram a última vez que leram a respeito, seguido dos que leram em tempo menor que 6 meses $(15,4 \%, n=4), 6$ meses a 2 anos $(30,8 \%, n=8)$ e acima de 2 anos $(7,7 \%, \mathrm{n}=2)$.

Tabela 3 - Conhecimento das normas de biossegurança aos de profissionais atuantes em Unidade de Terapia Intensiva. Teresina, 2019, $\mathrm{n}=26$.

\begin{tabular}{clccc}
\hline Variável & Categoria & $\mathbf{n}$ & $\mathbf{( \% )}$ & $\boldsymbol{p}$ valor \\
\hline & $<6$ meses & 4 & 15,4 & \\
& 6 meses a 2 anos & 8 & 30,8 & \\
& $>2$ anos & 2 & 7,7 & \\
Última vez que leu as normas & Não lembra & 6 & 23,1 & $0,03 *$ \\
& Nunca & 4 & 15,3 & \\
& Lê frequentemente & 2 & 7,7 & \\
& TOTAL & $\mathbf{2 6}$ & $\mathbf{1 0 0}$ & \\
\hline
\end{tabular}

Sim $\quad 18 \quad 69,2$

Normas são cobradas no hospital

$\begin{array}{lcc}\text { Não } & 8 & 30,8 \\ \text { TOTAL } & \mathbf{2 6} & \mathbf{1 0 0}\end{array}$

Legenda: N= Número de participantes; \%= percentual; *p valor estatisticamente significante (Teste de Wilcoxon). Fonte: Autores (2019). 
A análise dos desempenhos em uma escala de 0 a 10 no questionário sobre práticas de biossegurança é apresentada na Tabela 4, evidenciando uma maior média de acerto em condutas corretas por parte dos médicos $(8,3 \pm 0,0$ pontos, $\mathrm{p}=0,53)$, profissionais com mais de 10 anos de formação $(8,18 \pm 0,78$ pontos, $\mathrm{p}=0,22)$ e com até 2 anos de experiência profissional $(8,30 \pm 0,81$ pontos, $\mathrm{p}=0,11)$. Ao correlacionar as práticas de biossegurança com o tempo de trabalho no referido hospital, observa-se predominância para aqueles que atuam por mais de 3 anos na instituição $(8,13 \pm 0,74, p=0,002)$.

Tabela 4 - Associação entre perfil do profissional e média de desempenho em questionário sobre práticas de biossegurança em Unidade de Terapia Intensiva. Teresina, 2019, $\mathrm{n}=26$.

\begin{tabular}{|c|c|c|c|c|}
\hline Variável & Categoria & Média & DP & p valor \\
\hline \multirow[t]{4}{*}{ Profissão } & Médico & 8,3 & 0,00 & \multirow{4}{*}{0,53} \\
\hline & Enfermeiro & 7,6 & 0,90 & \\
\hline & Fisioterapeuta & 7,4 & 1,37 & \\
\hline & Técnico de enfermagem & 7,9 & 1,09 & \\
\hline \multirow[t]{3}{*}{ Tempo de formação (anos) } & Até 2 & 7,05 & 1,59 & \multirow{3}{*}{0,22} \\
\hline & 2 a 10 & 7,88 & 0,77 & \\
\hline & $>10$ & 8,18 & 0,78 & \\
\hline \multirow{3}{*}{$\begin{array}{l}\text { Tempo de experiência } \\
\text { profissional (anos) }\end{array}$} & Até 2 & 8,30 & 0,00 & \multirow{3}{*}{0,11} \\
\hline & Entre 2 a 3 & 6,64 & 1,66 & \\
\hline & $>3$ & 8,05 & 0,81 & \\
\hline \multirow{3}{*}{$\begin{array}{l}\text { Tempo de trabalho na } \\
\text { instituição (anos) }\end{array}$} & De 1 a 2 & 7,88 & 0,83 & \multirow{3}{*}{$0,002^{*}$} \\
\hline & Entre 2 e $3^{(a)}$ & 5,81 & 1,17 & \\
\hline & $>3^{(a)}$ & 8,13 & 0,74 & \\
\hline
\end{tabular}

$\mathrm{N}=$ Número de participantes; $\mathrm{DP}=$ Desvio padrão; ${ }^{*} p$ valor estatisticamente significante (Teste de Kruskal Wallis). ${ }^{(a)}$ diferença estatisticamente significante $(\mathrm{p}=0,03)$ na comparação entre duas categorias por teste de Student-Newman-Keuls. Fonte: Autores (2019).

\section{Discussão}

Apesar de todo suporte empregado na UTI, sabe-se que os pacientes criticamente enfermos são mais vulneráveis à infecção hospitalar, em comparação às demais unidades. O risco de infecção é diretamente proporcional à gravidade da doença, a natureza dos procedimentos, tempo de internação, mecanismos imunológicos, bem como o manejo dos profissionais de saúde, que devem ser pautados nas normas de biossegurança (Cabral \& Silva, 2013).

A promoção de saúde gerada na UTI é dada por uma equipe de profissionais de diversos campos de atuação, que se propõe enfatizar a importância da assistência aos pacientes internados, garantindo um atendimento integral e melhoria diante do quadro clínico apresentado (Evangelista et al., 2016). Ao analisar a equipe dos profissionais atuantes no referido hospital estadual, observa-se a multidisciplinariedade quanto a sua formação e com predomínio de profissionais experientes com mais de 3 anos de atuação. Semelhante ao perfil dos avaliados, Ribeiro (2016) ao observar 52 profissionais intensivistas atuantes em um hospital localizado no Distrito Federal mostrou predomínio dos profissionais técnicos de enfermagem (55,77\%), seguidos da enfermagem $(19,23 \%)$, medicina $(17,30 \%)$ e fisioterapia $(7,70 \%)$, e que a equipe é composta, em sua maioria, por profissionais mais experientes e do gênero feminino.

O ambiente hospitalar apesar de gerar suporte à saúde dos necessitados, é um local rico em microrganismos. A UTI destaca-se pelo maior risco de aquisição das infecções hospitalares, dada a disseminação bacteriana, frequente uso de antibióticos e contato profissional/paciente, fazendo necessário a proteção dos mesmos, no intuito de evitar a disseminação e a piora do quadro clínico dos pacientes criticamente enfermos, como dos próprios profissionais ali atuantes (Oliveira et al., 2009). Segundo Faria et al. (2019) dentro dos EPIs necessários ao uso na UTI, incluem-se: luvas, capote, propés, óculos e touca, e estes devem ser fornecidos pelos gestores da saúde, de acordo com o órgão competente.

Dentro desse contexto, os hospitais devem adotar medidas habituais de prevenção e controle de infecções, através das precauções padrão (Aguiar et al., 2008). Dentro da análise realizada a respeito da disponibilidade dos EPIs, os profissionais 
mostraram ter acesso a todos os EPIs, no entanto, com limitação quanto aos óculos e propés. Castro (2016) ao avaliar a infraestrutura hospitalar do Distrito Federal e as práticas de biossegurança dos seus profissionais de saúde da UTI, foi discutido sobre a disponibilidade dos EPIs na UTI, mostrando haver limitação de alguns destes, como avental, luvas, máscaras e óculos, o que concerne com os dados obtidos neste estudo. A indisponibilidade de EPIs impede que as intervenções sejam realizadas de forma segura, bem como o controle da disseminação das infecções, colocando em risco a integridade dos profissionais, com o quadro clínico dos pacientes.

Quanto ao uso de EPIs, os profissionais referiram, de modo geral, utilizá-los, no entanto, que estes não são empregados em sua completude em todas as intervenções, sendo algumas restritas apenas para as intervenções invasivas. Os dados obtidos corroboram com o estudo feito por Rieth et al. (2014) que evidenciou como se dá a utilização dos EPIs dos profissionais, no qual foram avaliados durante um mês em seu posto de trabalho, com base no uso de EPIs em todos os procedimentos feitos no trabalho, no qual obtiveram resposta positiva ao afirmarem que fazem uso de todos os EPIs disponíveis. A indisponibilidade dos EPIs é justificada pelas práticas realizadas, onde são classificadas em invasivas e não invasivas. Dado aos riscos apresentados nas manobras invasivas, há consenso entre os estudos a necessidade da total proteção ao profissional e paciente por meio do uso de todos os EPIs, como mostra o estudo de De Sousa et al. (2011), onde ao analisar o uso dos EPIs na UTI, mostra que o referido hospital promove incentivo ao uso, no entanto, que há predominância no uso dos EPIs em procedimentos não invasivos. $82 \%$ dos profissionais que compõem a equipe multidisciplinar atuantes na UTI descrevem receber os EPIs pelo referido hospital, no entanto, $22 \%$ descrevem usar às vezes, justificado pelas manobras invasivas que acabam expondo significantemente os profissionais, logo a maior predominância no uso dos mesmos.

Quando indagados sobre a frequência de troca da vestimenta hospitalar, os profissionais relataram ocorrer a trocar uma vez por cada plantão, referindo-se a entrada e saída do mesmo. Os entrevistados descrevem que não é fornecido uniforme para o uso nos plantões, bem como as vestimentas utilizadas por eles não são higienizadas pelo hospital, tendo os mesmos que levá-las para suas residências e realizarem a limpeza destas. Tal fato infringe consideravelmente à Norma Regulamentadora $\mathrm{n}^{\circ}$ 32, no qual consta que a higienização das vestimentas utilizadas nos centros cirúrgicos e obstétricos, serviços de tratamento intensivo, unidades de pacientes com doenças infectocontagiosa e com contato direto da vestimenta com material orgânico, deve ser de responsabilidade do empregador (Brasil, 2005; Oliveira et al., 2012).Segundo Boyce (2007) a principal via de transmissão de microrganismos ocorre por meio das mãos dos profissionais de saúde e pacientes, seguido do manejo de equipamentos e no vestuário dos profissionais (jaleco, avental e uniforme). Rice (2008) em seu estudo cita a alta prevalência de Staphylococcus aureus, Acinetobacter baumannii, Klebsiela pneumonia e Pseudomonas aureuginosa nos jalecos, aventais e uniforme, conduzindo a graves infecções, em especial aos pacientes da UTI, que são submetidos a inúmeras terapêuticas farmacológicas, incluindo antimicrobianos, aos quais muitos dos microrganismos tornam-se resistentes (Oliveira et al., 2012).

Ao serem questionados sobre a frequência da higienização das mãos, todos os entrevistados afirmaram realizar a adequada antissepsia das mãos antes e após de todos os procedimentos realizados. Contudo, na observação feita, a higienização não ocorreu como descrito, sendo identificado que alguns profissionais após a intervenção apenas retiravam as luvas e direcionavam-se ao preenchimento de prontuários. Concomitante as observações realizadas no referido estudo, Souza et al. (2015) descrevem que a razão entre número de manejo ao paciente e o número de higienização das mãos teve taxa de adesão de 43,7\%, que pode ser considerada insatisfatória, apontando o risco de transmissão de infecção hospitalar.

A higienização das mãos é considerada a ação isolada mais importante no controle de infecções em serviços de saúde. Quando se lava as mãos, utilizando a técnica correta, eliminam-se bactérias da microbiota transitória e parte da residente, removendo microrganismos, células mortas, sujidades e oleosidade da pele (Souza et al., 2011). Segundo Martinez et al. (2009) a lavagem das mãos é indicada antes de ministrar medicamentos por via oral e preparar a nebulização, antes e após a realização de trabalhos hospitalares, atos e funções fisiológicas ou pessoais, antes e depois do manuseio de cada paciente, do preparo de 
materiais ou equipamentos, da coleta de espécimes, da aplicação de medicamentos injetáveis e da higienização e troca de roupa dos pacientes.

De acordo com a NR 32 a lavagem das mãos deve acontecer no ambiente de trabalho com lavatório exclusivo provido de água corrente, sabonete líquido, toalha descartável e lixeira com sistema de abertura sem contato manual, de forma que a lavagem das mãos deverá acontecer no mínimo antes e depois da utilização de luvas, sendo que estas, não devem substituir o processo de lavagem (Brasil, 2005) Quando observado o local de higienização das mãos do referido hospital, não cumpriam com as normas citadas pela NR 32, com destaque para a torneira, na qual se fazia necessário o contato manual para sua abertura e fechamento, propiciando o processo de contaminação.

Para que as normas de biossegurança sejam efetivadas, faz-se necessário que os profissionais tenham o conhecimento teórico-prático destas. Sabe-se que dada a dimensão de formação profissional na área da saúde, alguns cursos apresentam em sua grade curricular disciplinas voltadas para conhecimento destas, no entanto, isso não é comum a todas as formações. Trevisan et al. (2013) entrevistaram 29 profissionais, mostrando que 20,69\% afirmaram não conhecer a aplicação da biossegurança no seu local de trabalho e os outros 79,31\% ter conhecimento dessa aplicabilidade, porém, declararam que a instituição não fornece treinamento para os possíveis riscos da não utilização dos EPIs.

Apesar dos profissionais avaliados afirmarem que o hospital cobra as práticas de biossegurança, bem como terem conhecimento das normas e que as realizam em suas intervenções, ao analisar o desempenho destes apenas os médicos conseguiram pontuar satisfatoriamente. Embora esse perfil de profissional tenha atingido maior média, tal diferença não foi significativa, evidenciando que profissão, tempo de formação e tempo de experiência profissional não tem associação com melhor adesão e conhecimento as condutas corretas em UTI. Passos et al. (2013) observaram 20 profissionais a respeito das práticas de biossegurança, sendo os médicos os profissionais que menos cometeram infrações, seguidos dos enfermeiros, fisioterapeutas e técnicos de enfermagem. Acredita-se que os médicos apresentaram menor índice de infrações dado ao número reduzido destes profissionais na UTI, além dos poucos procedimentos práticos e de risco que os mesmos têm que desempenhar em comparação com as outras categorias profissionais.

Concomitante ao desempenho dos profissionais, não foram identificadas as normas de biossegurança propostas pelo hospital expostas no ambiente hospitalar, sendo identificadas apenas as instruções do passo a passo da higienização das mãos próximo às duas pias disponibilizadas. Além disso, observa-se que o conhecimento das normas, sobre o ponto de vista dos profissionais, mostra-se desatualizada e/ou inadequada, uma vez que a minoria afirma ler com frequência, contendo aqueles que relatam nunca terem lido. Segundo Cararro et al. (2012) o conhecimento das normas de biosseguranças deve ser implantado na formação acadêmica dos profissionais e que isso não exclui o papel dos gestores em saúde na capacitação dos profissionais, uma vez que os processos de cuidado sofrem mudanças com frequência, necessitando que os saberes sejam constantemente reciclados.

As observações realizadas neste estudo, bem como, a aplicação do questionário limitou-se a apenas um turno e aos profissionais que se mantiveram no mesmo, buscando assim haver reprodutividade dos dados apurados, acredita-se que expandir a percepção das demais equipes multidisciplinares atuantes na UTI nos demais turnos possibilitará uma análise mais apurada e diversificada do perfil dos profissionais quanto às normas de biossegurança aplicadas. Logo, este trabalho traz a reflexão sobre a análise das práticas profissionais nas UTIs, visando novos estudos dentro da temática como forma de promover a conscientização, bem como a educação em saúde no âmbito hospitalar, não se limitando apenas às UTIs.

\section{Conclusão}

Os profissionais de saúde afirmam possuir conhecimento das normas de biossegurança, no entanto, houve divergência dos resultados obtidos no questionário com as observações feitas no que tange ao cumprimento destas. 
Percebe-se a necessidade da atenção quanto às práticas de biossegurança empregada pelos profissionais no ambiente de trabalho. Por mais que haja conhecimento da existência das mesmas e da importância do seu emprego nas intervenções, estas são negligenciadas, o que cursa em um elevado risco de contaminação aos próprios profissionais de saúde e aos pacientes, o que sugere a necessidade de uma fiscalização mais rigorosa.

\section{Referências}

Aguiar, D. F. de, Lima, A. B. G., \& Santos, R. B. (2008). Uso das precauções-padrão na assistência de enfermagem: um estudo retrospectivo. Escola Anna Nery, 12(3), 571-576. https://doi.org/10.1590/S1414-81452008000300027

Almeida, A. B. de S., \& Albuquerque, M. B. M. de. (2000). Biossegurança: um enfoque histórico através da história oral. História, Ciências, SaúdeManguinhos, 7(1), 171-184. https://doi.org/10.1590/S0104-59702000000200009

Anvisa, A. N. de V. S. (2005). Biossegurança. Revista Saúde Pública, 39(6), 989-991.

Barros, L.L. dos S., Maia, C. do S. F., \& Monteiro, M. C. (2016). Fatores de risco associados ao agravamento de sepse em pacientes em Unidade de Terapia Intensiva. Cadernos Saúde Coletiva, 24(4), 388-396. https://doi.org/10.1590/1414-462x201600040091

Boyce, J. M. (2007). Environmental contamination makes an important contribution to hospital infection. J Hosp Infect. 65(2),50-

4. https://doi.org/10.1016/S0195-6701(07)60015-2

Brasil. (2005). Portaria TEM n 485, de 11 de novembro de 2005. NR 32 - Segurança e saúde no trabalho em serviços de saúde. Diário Oficial da República Federativa do Brasil. Guia Trabalhista.

Cabral, F. W., \& Silva, M. Z. O. (2013). Prevenção e controle de infecções no ambiente hospitalar. Sanare, 12(1), 59-70.

Cararro, T.E., Gelbcke, F. L., Sebold, L. F., Kempfer, S. S., Zapelini, M. C., \& Waterkemper, R. (2012). A biossegurança e segurança do paciente na visão de acadêmicos de enfermagem. Revista Gaúcha de Enfermagem, 33(3), 14-19. https://doi.org/10.1590/S1983-14472012000300002

Castro, A. F. DE. (2016). Práticas de precauções em Unidade de Terapia Intensiva de um hospital de ensino do Distrito Federal.

Correa, C. F., \& Donato, M. (2007). Biossegurança em uma unidade de terapia intensiva: a percepção da equipe de enfermagem. Escola Anna Nery, 11(2), 197-204. https://doi.org/10.1590/S1414-81452007000200003

De Souza, E. L. V., Do Nascimento, J. C., Caetano, J. A., \& Ribeiro, R. C. V. (2011). Uso dos equipamentos de proteção individual em unidade de terapia intensiva. Revista de Enfermagem de Referência, 4, 125-133.

Evangelista, V. C., Domingos, T. da S., Siqueira, F. P. C., \& Braga, E. M. (2016). Equipe multiprofissional de terapia intensiva: humanização e fragmentação do processo de trabalho. Revista Brasileira de Enfermagem, 69(6), 1099-1107. https://doi.org/10.1590/0034-7167-2016-0221

Faria, L. B. G. de, Santos, C. T. B. dos, Faustino, A. M., Oliveira, L. M. de A. C., \& Cruz, K. C. T. da. (2019). Knowledge And Adherence Of The Nurse To Standard Precautions In Critical Units. Texto \& Contexto - Enfermagem, 28. https://doi.org/10.1590/1980-265x-tce-2018-0144

Martinez, M. R., Campos, L. A. A. F., \& Nogueira, P. C. K. (2009). Adesão à técnica de lavagem de mãos em Unidade de Terapia Intensiva Neonatal. Rev Paul Pediatr, 27(2), 179-85. https://doi.org/10.1590/S0103-05822009000200010

Nishide, V. M., Benatti, M. C. C., \& Alexandre, N. M. C. (2004). Ocorrência de acidente do trabalho em uma unidade de terapia intensiva. Revista LatinoAmericana de Enfermagem, 12(2), 204-211. https://doi.org/10.1590/S0104-11692004000200009

Oliveira, A. C. de, Cardoso, C. S., \& Mascarenhas, D. (2009). Intensive care unit professionals' knowledge and behavior related to the adoption of contact precautions. Revista Latino-Americana de Enfermagem, 17(5), 625-631. https://doi.org/10.1590/S0104-11692009000500005

Oliveira, A. C., Silva, M. D. M., \& Garbaccio, J. L. (2012). Vestuário de profissionais de saúde como potenciais reservatórios de microrganismos: uma revisão integrativa. Texto Contexto Enferm., 21(3), 684-91. https://doi.org/10.1590/S0104-07072012000300025

Passos, B. B. C., Vasconcelos, T. B. de, Bastos, V. P. D., \& Sousa, C. T. de. (2013). Desatenção às Normas de Biossegurança por Profissionais de Saúde em Unidade de Terapia Intensiva de Hospital na Cidade de Fortaleza/CE. Revista de Saúde Pública de Santa Catarina, 6(1).

Pinto, A. M., Júnior, A. de F. S., \& Júnior, A. F. R. S. (2005). Emprego Do Plano-Sequência No Gerenciamento Da Roupa Hospitalar: Avaliação De Aspectos De Biossegurança. Revista Prática Hospitalar, 7I(38), 1-7.

Ribeiro, L. S. (2016). Adesão dos profissionais de unidade de terapia intensiva à pratica de higiene das mãos: avaliação à luz da estratégia multimodal da Organização Mundial de Saúde.

Rice, L; B. (2008). Editorial commentary. Federal funding for the study of antimicrobial resistance in nosocomial pathogens: No ESKAPE. J Infect Dis., 197(8), 1079-81. https://doi.org/10.1086/533452

Rieth, G. H., Loro, M. M., Stumm, E. M. F., Rosanelli, C. L. S. P., Kolankiewicz, A. C. B., \& Gomes, J. S. (2014). Uso de equipamentos de proteção individual pela enfermagem em uma unidade de emergência hospitalar. Revista de Enfermagem Da UFPE On-Line, 8(2), 365-371. https://doi.org/10.5205/reuol.4688-38583-1-RV.0802201418 
Research, Society and Development, v. 10, n. 5, e1010514613, 2021

(CC BY 4.0) | ISSN 2525-3409 | DOI: http://dx.doi.org/10.33448/rsd-v10i5.14613

Santuzzi, C. H., Scardua, M. J., Reetz, J. B., Firme, K. S., Lira, N. O., \& Gonçalves, W. L. S. (2013). Aspectos éticos e humanizados da fisioterapia na UTI: uma revisão sistemática. Fisioterapia Em Movimento, 26(2), 415-422. https://doi.org/10.1590/S0103-51502013000200019

Silva, J. V. F. da, \& Rodrigues, A. P. R. A. (2015). A infecção nosocomial em unidade de terapia intensiva neonatal. Caderno de Graduação - Ciências Biológicas e Da Saúde - UNIT - ALAGOAS, 3(1 SE-Artigos), 129-138. https://periodicos.set.edu.br/fitsbiosaude/article/view/2633

Souza, E. L. V. de, Nascimento, J. C. do, Caetano, J. A., \& Enfermeira, R. C. V. R. (2011). Uso dos equipamentos de proteção individual em unidade de terapia intensiva. Revista de Enfermagem RFeferência, serIII, 125-133. http://www.scielo.mec.pt/scielo.php?script=sci_arttext\&pid=S0874-

$02832011000200013 \&$ nrm $=$ isso

Souza, L.M., Ramos, M. F., Becker, E. S. S., Meirelles, L. C. S., \& Monteiro, S. A. O. (2015). Adesão dos profissionais de terapia intensiva aos cinco momentos da higienização das mãos. Rev Gaúcha Enferm, 36(4), 21-8. https://doi.org/10.1590/1983-1447.2015.04.49090

Talhaferro, B., Barboza, D. B., \& Oliveira, A. R. De. (2008). Adesão ao uso dos equipamentos de proteção individual pela enfermagem. Rev. Ciênc. Méd., (Campinas), 17(3-6), 157-166.

Trevisan, M., Rosa, C., Lima, C., \& Sousa, J. (2013). The importance of applied to bio-security professional radiology. Rev Elet Gestão \& Saúde, 4(3), 786800 .

World Health Organization. (2020). Rational use of personal protective equipment for coronavirus disease (COVID-19): interim guidance, 27 February 2020. World Health Organization. https://apps.who.int/iris/handle/10665/331215 